\title{
SUSY model and dark matter determination in the compressed-spectrum region at the ILC
}

Mikael Berggren*†

DESY, 22603 Hamburg, Germany

E-mail: mikael.berggren@dey. de

It is an appealing possibility that the observed dark matter density in the universe can be fully explained by SUSY. The current experimental knowledge indicates that this possibility strongly favors a co-annihilation scenario. In such scenarios, the mass difference between the next-tolightest SUSY particle (the NLSP) and the lightest one (the LSP) is quite small, which assures that the annihilation cross-section is sufficient not to predict a too large abundance of dark matter. However, the small mass difference also means that observing SUSY becomes hard at hadron colliders, where the observation hinges on the tell-tale signature of missing transverse energy: if the mass difference NLSP-to-LSP is small, only little energy is carried away by the invisible LSP. This is also true even if several other SUSY particles are within the kinematic reach, since these states would to a large extent decay via cascades ending with an NLSP to LSP decay. A lepton collider does not have this problem. The clean environment and known initial state at such machines assures that SUSY can be detected even if the mass difference is very small, provided the center-of-mass energy is sufficiently high. We present prospects for observation and precision characterization of SUSY with small mass differences at the ILC, based on detailed simulations of the ILD detector concept. The resulting possibility to predict the dark matter relic density is evaluated and compared to the precision obtained from the Planck mission. Taking a specific model as an example, we also discuss the synergies from combining ILC and HL-LHC results.

38th International Conference on High Energy Physics

3-10 August 2016

Chicago, USA

* Speaker.

${ }^{\dagger}$ On behalf of the ILC Physics and Detector Study. 


\section{Introduction}

Many observations indicate that a large fraction of the matter in the universe is dark, i.e. not visible in observations of electromagnetic radiation. The data on the temperature power spectrum of the cosmic microwave background (CMB) and its polarisation obtained by the Planck mission give the most precise cosmological determination of the dark matter (DM) relic density $\Omega_{C D M}$, and is $\Omega_{C D M}=0.1197 \pm 0.0022$ [1]. If such an abundance of DM in the early universe is to be explained by the existence of hitherto unknown elementary particle, one needs a balance between early universe production and decay of this particle. One finds that this could be obtained by a weakly interacting, stable massive particle (a WIMP). Since the lightest Super-symmetric particle (the LSP) has such properties, it is quite compelling to assume that it is the DM. However, if the LSP is the only SUSY particle in the mass-range needed, it would tend to be too abundant. Therefore, other SUSY particles are expected to have been close in density in the hot early universe, i.e. to be close to mass-degenerate with the LSP. Such scenarios are called a coannihilation scenarios, and the coannihilation next-to-lightest SUSY particle (the NLSP) could be the $\tilde{\tau}_{1}$, the $\tilde{\mathfrak{t}}_{1}$ or the $\tilde{\chi}_{1}^{ \pm}$. Such scenarios are also compelling for other reasons. If the totality of current observations sensitive to SUSY are fitted to a general SUSY model with 10 free parameters, as has been done by the Mastercode collaboration[2], one finds that models where the bosino and slepton-sector is quite compressed fits the observations best.

In this contribution, we study in particular the $\tilde{\tau}$ coannihilation scenario at the ILC. Using the micrOMEGAs code[3], it has been shown [4] that a $1 \%$ variation of $M_{\tilde{\tau}}$ or $M_{\tilde{\chi}_{1}^{0}}$ changes the predicted relic abundance by $5 \%$, while a similar variation of the $\tilde{\tau}$ mixing angle $\left(\theta_{\tilde{\tau}}\right)$ or the binoness of the LSP $\left(N_{11}\right)$ changes the abundance by $1 \%$ and $3.5 \%$, respectively. Therefore, to match the $2 \%$ uncertainty on the relic abundance obtained by Planck one needs per mil-level precision measurements of the LSP and NLSP masses, and percent-level precision on the LSP and NLSP mixings. In Section 2, we introduce the ILC and point out important differences to the LHC for the study of the $\tilde{\tau}$ coannihilation scenario. Section 3 presents the results from our study on the prospects to measure the key-parameters of the model at the ILC, and comment on the LHC prospects.

\section{Compressed spectra at the ILC and the LHC}

The International Linear Collider (ILC) [5] is a proposed $34 \mathrm{~km}$ long $e^{+} e^{-}$collider, colliding electrons and positrons accelerated by two separate superconducting linear accelerators. The centre-of-mass energy $\left(E_{C M S}\right)$ is to be tunable between 200 and $500 \mathrm{GeV}$, with an upgrade path to $1 \mathrm{TeV}$ defined. The electron and positron beams are polarised to $80 \%$ and $>30 \%$, respectively ${ }^{1}$. Once it is tuned up, the machine will deliver an integrated luminosity of $290 \mathrm{fb}^{-1}$ per year at $E_{C M S}=500 \mathrm{GeV}$.

In contrast to proton colliders, it is a collider of fundamental particles, meaning that the initial state is known, and that there is no spectator event accompanying the hard interaction. Furthermore, the production processes are electroweak, which implies lower event-rates. The detectors can be

\footnotetext{
${ }^{1}$ In the following, $\mathscr{P}_{p^{-}, p^{+}}$denotes the beam-polarisation configuration $\mathscr{P}\left(e^{-}, e^{+}\right)=\left(p^{-}, p^{+}\right)$, with $p^{-}$and $p^{+}$ given in percent.
} 
made much thinner, since they are not required to be radiation-hard. They can be as close as 1 to $2 \mathrm{~cm}$ to the interaction-point, and can have very close to $4 \pi$ coverage. In addition, the low rates means that the detectors can register all interactions, i.e. there is no need for any triggering. In the coannihilation scenario, both the excellent hermeticity and the trigger-less operation are required features be able to detect NLSP-pair production events above background from $\gamma \gamma$ events. Further details of the proposed detectors for the ILC can be found in [6].

LHC puts strong limits on the mass of first and second generation squarks and on the gluino. These states have no direct influence on DM, the muon magnetic moment anomaly, nor naturalness. If one requires GUT-scale unification of the SUSY mass-parameters, the limits do indirectly constrain the states that are relevant, i.e. the third generation squarks and the EW sector. By removing this requirement ${ }^{2}$, the LHC limits no longer constrain SUSY contributions to the precision observables and DM. If, in addition, the SUSY spectrum is compressed, one would expect long decay-cascades at the LHC, ending up at the NLSP, which decays into the LSP and, due to the low mass difference required in coannihilation models, to a soft visible system. This is thus not a model predicting large missing $\mathrm{E}_{T}$, nor a simplified model where the produced heavy coloured particles decay directly to the LSP. Taken together, the current limits on coannihilation models from LHC become significantly weaker than those obtained from simplified models or large missing $\mathrm{E}_{T}$ ones.

\section{The Stau-coannihilation STCx models}

A SUSY coannihilation model should - in addition to containing a DM candidate - accommodate the LHC limits, the requirement from naturalness that the third generation squarks should be comparatively light, and that the higgs-sector, in particular the higgs-mass, should agree with observations. It should also fulfil all constraints for precision measurements. We found that this can all be achieved in a set of SUSY models with 12 parameters [7], called STCx, which we study in the following. The different models (STC4 to STC8) differ only in the magnitude of the $\tilde{\mathrm{t}}$ mass, while the bosino and slepton sectors are identical. Figure 1(a) shows the mass-spectrum of the STC8 model. In [8], the models were studied at LHC14 and ILC with the fast detector simulation programs. The ILC study is the topic of the next sections, while a summary of the findings at LHC is that the high mass first and second generation squarks and the gluino will not be seen even at the HL-LHC, since the cross-section is quite small. However, the lower mass third generation squarks will eventually be discovered, and the bosino/slepton sector will be detected, but with little possibility to disentangle the different states.

\subsection{STC at ILC}

By inspecting figure 1, one sees that the expected signal at a $500 \mathrm{GeV}$ ILC typically contains a few leptons and LSP:s, leading to low multiplicity events. The events are central in the detector, and would have much missing energy. Furthermore, the cross-sections might be close to $1 \mathrm{pb}$ for certain channels. Heavier states often decays in cascades over the NLSP, which is the $\tilde{\tau}_{1}$. The mass difference between the $\tilde{\tau}_{1}$ and the LSP is $\sim 10 \mathrm{GeV}$, meaning that the energy of the $\tau$ :s from decays of pair-produced $\tilde{\tau}_{1}: \mathrm{s}$ is between 2.5 and $45 \mathrm{GeV}$. The SM background to such events is

\footnotetext{
${ }^{2}$ The couplings at the GUT scale can still be required to unify.
} 


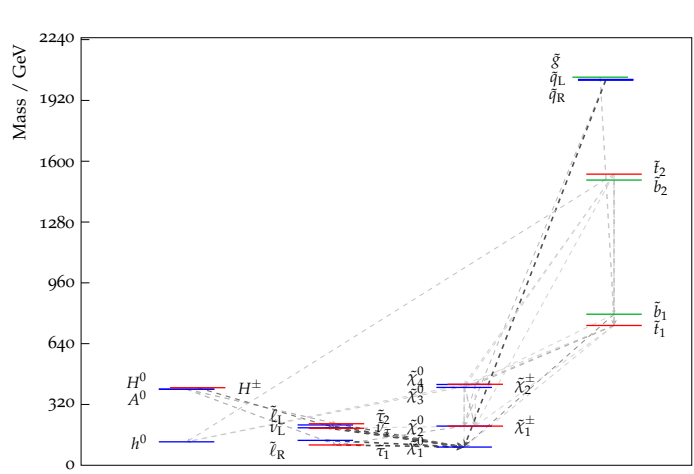

(a)

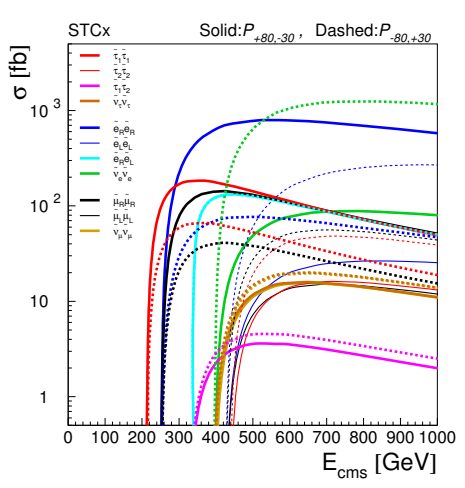

(b)

Figure 1: (a): Mass-spectrum and decay modes (with BR $>10 \%$ ) of STC8. Heavier lines indicate larger BR; (b): production cross-sections at ILC for sfermions, for $\mathscr{P}_{+80,-30}$ (solid) and $\mathscr{P}_{-80,+30}$ (dashed).

either processes with real missing energy, e.g. $Z Z, W W \rightarrow \ell \ell v v$, or with "fake" missing energy e.g. $\gamma \gamma$ processes, ISR, or single IVB, where a high energy final-state particle escapes the detector through the low angle acceptance holes due to the beam-pipes.

These considerations lead to the following selection criteria, valid for all slepton studies: The events should contain less than 10 charged particles, and two opposite charge lepton candidates (electrons, muons, or $\tau$-decay products). The total visible energy in the event, $E_{\mathrm{vis}}$ and the missing mass, $M_{\text {miss }}$, should be $<300 \mathrm{GeV}$ and $>200 \mathrm{GeV}$, respectively, and all particles should have momentum $<180 \mathrm{GeV}$.

An integrated luminosity of $1000 \mathrm{fb}^{-1}$ at $E_{c m s}=500 \mathrm{GeV}$, evenly divided between beam polarisations of $\mathscr{P}_{+80,-30}$ and $\mathscr{P}_{-80,+30}$ was assumed. Both signal and background were simulated with the Whizard V1.95 [9] event-generator. The response of the ILD detector [6] was simulated with the fast simulation program SGV [10].

To extract the masses of the SUSY particles, one uses that in the lab-frame, the highest and lowest possible lepton energies are

$$
E_{(\min )}^{\prime}=\left(\gamma_{-)}^{+} \gamma \beta\right) E_{\text {rest frame }}=\frac{E_{\text {Beam }}}{2}\left(1-\left(\frac{M_{\tilde{\chi}_{1}^{0}}}{M_{\tilde{\ell}}}\right)^{2}\right)\left(1_{(-)}^{+} \sqrt{1-\left(\frac{M_{\tilde{\ell}}}{E_{\text {Beam }}}\right)^{2}}\right)
$$

In this expression, there are two observables $\left(E_{\min }^{\prime}\right)$ and two parameters $\left(M_{\tilde{\ell}}\right.$ and $\left.M_{\tilde{\chi}_{1}^{0}}\right)$. For $\tilde{e}_{\mathrm{R}}$ and $\tilde{\mu}_{\mathrm{R}}, E_{\min }^{\prime}$ can be measured very well at the ILC, while only the end-point of the spectrum of $E_{\tau-j e t}$, which is equal to $E_{\max }^{\prime}$, can be well measured for $\tilde{\tau}_{1}$. Therefore, we use $\tilde{\mathrm{e}}_{\mathrm{R}}$ and $\tilde{\mu}_{\mathrm{R}}$ to determine $M_{\tilde{\chi}_{1}^{0}}$, and the end-point of the $E_{\tau-\text { jet }}$ spectrum to determine $M_{\tilde{\tau}_{1}}$.

\subsection{The selectron, the smuon and the LSP mass}

As can be seen in Fig. 1 (b), the slepton pair production cross sections are large in our scenario $^{3}$. Very precise measurements of the slepton and LSP masses can be obtained from the edges

\footnotetext{
${ }^{3}$ Due to the $t$-channel neutralino exchange, it is particularly large for the selectron channel.
} 
of the lepton spectra. In addition to the general slepton selection criteria, it is demanded that the two lepton candidates have the same flavour. To separate the right-handed and left-handed states, switching of the beam-polarisation is helpful, as it will preferentially select the process either with left-handed or right-handed slepton production. The left-handed states are heavier than than righthanded ones, meaning that the different kinematics can be used to further enhance the separation. Finally, a "Tag and probe" technique is used: a lepton-candidate is accepted only if the other candidate in the event has a momentum within the kinematic limits of the process. With these cuts, the selection efficiency is $51 \%$ for $\tilde{\mathrm{e}}_{\mathrm{R}}$, while it is slightly higher, $65 \%$ for $\tilde{\mu}_{\mathrm{R}}$.

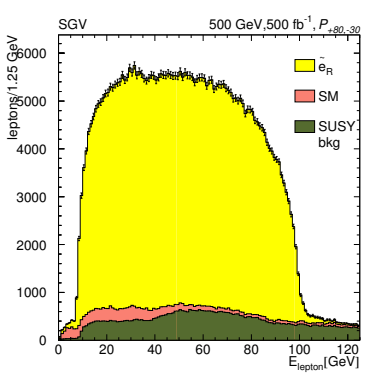

(a)

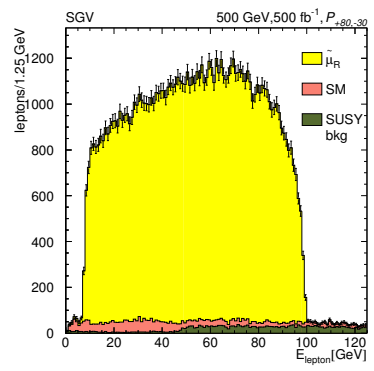

(b)

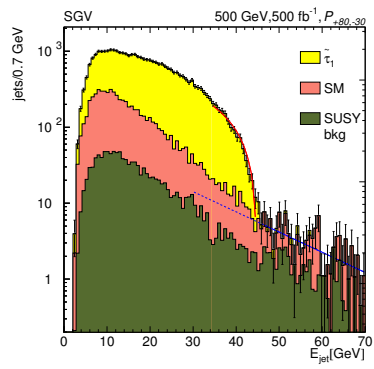

(c)

Figure 2: Spectra of electron (a), muon (b) and $\tau$-jet (c) energies in selected di-leptons events after collecting $500 \mathrm{fb}^{-1}$ of data for beam-polarisation $\mathscr{P}_{+80,-30}$.

Figure 2 (a) and (b) shows the spectra in selected di-leptons events. The signal stands out above quite small SM and SUSY background. By fitting the two edges in each spectrum, and using eq. (3.1), we obtain $M_{\tilde{\chi}_{1}^{0}}=95.47 \pm 0.16 \mathrm{GeV}$ and $M_{\tilde{\mathrm{e}}_{\mathrm{R}}}=126.20 \pm 0.21 \mathrm{GeV}$ from the $\tilde{\mathrm{e}}_{\mathrm{R}}$ spectrum, and $M_{\tilde{\chi}_{1}^{0}}=95.47 \pm 0.38 \mathrm{GeV}$ and $M_{\tilde{\mu}_{\mathrm{R}}}=126.10 \pm 0.51 \mathrm{GeV}$ from the $\tilde{\mu}_{\mathrm{R}}$ spectrum. This is in good agreement with the model-values: the true masses in STCx are $M_{\tilde{\chi}_{1}^{0}}=95.59 \mathrm{GeV}$, $M_{\tilde{\mathrm{e}}_{\mathrm{R}}}=126.24 \mathrm{GeV}$ and $M_{\tilde{\mu}_{\mathrm{R}}}=126.16 \mathrm{GeV}$. Combining the measurement of the LSP mass from these two analyses gives an uncertainty of $\sigma_{M_{\tilde{\chi}_{1}^{0}}}=147 \mathrm{MeV}$, i.e. slightly above 1 per mil.

\subsection{The stau mass and the mixings}

As was pointed out above, a precise determination of the $\tilde{\tau}$ sector is essential to test whether the $\tilde{\chi}_{1}^{0}$ is the main constituent of DM. Since the $\tilde{\tau}$ mass difference to the LSP is much smaller, and the $\tau$ decays before detection, the spectrum is softer than that of the leptons from $\tilde{\mathrm{e}}_{\mathrm{R}}$ or $\tilde{\mu}_{R}$ decays. In addition, also due to the decay of the $\tau$ :s, particle identification becomes less effective in suppressing the background. The signal therefore resembles $\gamma \gamma$ to a much larger extent, and also resembles $W W$ or $Z Z$ events decaying to $\tau \nu \tau \nu$. Several further criteria must therefore be added: The requirements on $E_{\mathrm{vis}}$ and $M_{\text {miss }}$ are strengthened to $<120 \mathrm{GeV}$ and $>250 \mathrm{GeV}$, respectively, and the visible mass should be $5 \mathrm{GeV}$ below $M_{Z}$, To further reduce the di-boson background, as well as the SUSY background from $\tilde{e}_{R^{-}}$or $\tilde{\mu}_{R}$-pair production, it was demanded that the candidate events are not same-flavour lepton ones. To reduce the $\gamma \gamma$ background, the direction of the missing momentum is required to be more central $\left(\left|\cos \theta_{\text {miss }}\right|<0.8\right), M_{\text {vis }}$ to be above $20 \mathrm{GeV}$, and the total energy observed below 30 degrees to the beam-axis to be below 2 
$\mathrm{GeV}$. A cut on the likelihood that the event is a $\gamma \gamma$ event is also imposed. With these cuts, the selection efficiency for $\tilde{\tau}_{1}$-pair production is $17 \%$.

The end-point of the spectrum of the $\tau$ decay-products is determined by fitting the background in the signal-free region well above the endpoint, and then fitting the excess above the extrapolated background fit to a signal contribution. Figure 2 (c) shows the energy-spectrum of selected $\tau$ jets. The endpoint could be determined to be $\mathrm{E}_{\text {endpoint }}=44.49_{-0.09}^{+0.11} \mathrm{GeV}$, corresponding to an uncertainty on $M_{\tilde{\tau}_{1}}$ of $200 \mathrm{MeV}$, if an uncertainty on the LSP mass from the ẽ and $\tilde{\mu}$ analysis of $\sim$ $100 \mathrm{MeV}$ is assumed. Hence, also $M_{\tilde{\tau}_{1}}$ can be determined to slightly above 1 per mil.

In [11], where a model quite similar to STCx has been studied, it is found that, in addition to $M_{\tilde{\tau}_{1}}$, the cross section can be determined at the level of $4 \%$, and the polarisation of $\tau$-leptons from the $\tilde{\tau}_{1}$ decay, which gives access to the $\tilde{\tau}$ and $\tilde{\chi}_{1}^{0}$ mixing, could be measured with an accuracy of $5 \%$. For the current study, the corresponding analysis is work-in-progress, but it seems quite probable that also mixings would be measurable at the required percent level.

\section{Conclusions}

SUSY models with a rich and compressed spectrum are still the best fit to data. They are not excluded by LHC. It is likely that LHC would discover such a model in the next few years, if it is realised in nature. In such models, a rich spectrum is reachable by the ILC, and ILC will be able to corroborate on any discovery made at LHC. In particular, ILC will be able to prove that the New Physics discovered at LHC is SUSY. Masses will be determined at per mil-level, mixings (probably) at percent-level. With such precisions, ILC will be capable to measure DM properties with an accuracy close to the cosmological observations on CMB from the Planck satellite, and hence to determine if indeed the LSP is the main contributor to DM.

\section{References}

[1] Planck Collaboration, P. A. R. Ade et al., Astron. Astrophys. 594 (2016) A13 [arXiv:1502. 01589 [astro-ph. CO]].

[2] K. J. de Vries et al., Eur. Phys. J. C 75 (2015) no.9, 422 [arXiv:1504.03260 [hep-ph] ].

[3] G. Belanger et al., Comput. Phys. Commun. 176 (2007) 367-382, [arXiv: hep-ph/ 0607059 ].

[4] S. L. Lehtinen, M. Berggren and J. List, arXiv:1602.08439 [hep-ph]

[5] C. Adolphsen, M. Barone, B. Barish, K. Buesser, P. Burrows, et al., ISBN 978-3-935702-77-5. [arXiv:1306.6328 [physics.acc-ph]].

[6] T. Behnke, J. E. Brau, P. N. Burrows, J. Fuster, M. Peskin, et al., ISBN 978-3-935702-78-2. [arXiv:1306.6329 [physics.ins-det]].

[7] H. Baer and J. List, Phys. Rev. D88 (2013) 055004, [arXiv:1307.0782 [hep-ph] ].

[8] M. Berggren et al., Eur. Phys. J. C 76 (2016) no.4, 183 [arXiv:1508.04383 [hep-ph] ].

[9] W. Kilian, T. Ohl, and J. Reuter, Eur.Phys.J. C71 (2011) 1742, [arXiv:0708.4233 [hep-ph]].

[10] M. Berggren, in International Workshop on Future Linear Colliders (LCWS11) Granada, Spain, September 26-30, 2011. 2012. [arXiv:1203.0217 [physics.ins-det]].

[11] P. Bechtle et al., Phys. Rev. D82 (2010) 055016, [arXiv:0908.0876 [hep-ex] ]. 